\title{
The "Sustainable Destination Structure" for Sustainable Tourist Demand - Selective Marketing for Sustainable Tourism Demand for Unpolluted Areas
}

\author{
Ines Ana Maria Jaho \\ PHD Candidate, "Aleksander Moisiu" University" Faculty of Business \\ Osman Metalla \\ Associated Professor, "Aleksander Moisiu" University" \\ Faculty of Professional Studies
}

\section{Abstract}

Observing the recent developments of the demand for tourist products, we notice some very important changes in the tourists' preferences of consumption toward a different sense of quality, asking for new quality models based on virginity, pure nature, highly maintained clean spaces, authenticity, cultural heritage and high responsibility and consciousness on sustainability. The natural environment represents the main resource to this demand on many tourism destinations. This is related to the fact that tourists increasingly are interested in spending their holidays in unspoiled natural territories. To this end, destination managers recently are under increased pressure to improve their eco-quality, maximize the hygiene, as well as to implement ecologically sustainable practices and systems. Based and stimulated by this evidence, a process of selective targeting/segmentation of tourist market could be an approach to sustainable destination management, both generally in the international market, but very promising to the Albanian case of the tourist sector future development, focusing at the region of Elbasan. Considering and analyzing the ecological footprint of Albania, and particularly that of Elbasan region, this paper will try to test these possibilities. To observe the feasibility of this approach, the study will be focused on tourists, between Albanians and foreign visitors, regarding their main reasons of returning in the same destinations. The questions to be treated will be mainly focused on the quality of the tourist services, the environmentally friendly behavior, as well as psychographic, behavioral, and socio-demographic personal characteristics of the tourists. Focusing and deepening in sustainable tourism destinations' management could foster the increase in the number of day-vacations for one year, strongly also influencing the normal 
development of the supporting industries. This asks for techniques which focus on eco-tourism and sustainability at the destinations, even why the tourist himself generally may not necessarily be interested in protecting and caring to the local environment. In conclusion, the study confirms the increasing trend of the orientation of the tourist demand toward unpolluted destinations and attractions, as well as the tendency to safeguard the environment and to use sustainable tourist resources

Keywords: Sustainability, Ecological Footprint, Tourism

\section{Introduction}

\section{Literature review}

According to the World Travel and Tourism Council (2019), world tourism contribution to the gross domestic product (GDP) is about $\$ 2.9$ trillion generating more than 334 million jobs, almost $10.6 \%$ of the total employment and $\$ 642^{1}$ billion of new capital investment invested only by 10 countries (USA, China, India, France, Japan, Germany, UK, Saudi Arabia, Spain, and Brazil) ${ }^{2}$. These figures give us a clear picture about the significance and the ability of tourism industry to contribute in the change of the worlds' and regional economy.

However, this enormous contribution and unplanned growth of tourism has its own impact to the recent changes of the world environment which is directly affected by the policies of tourism businesses and tourist managers. These undesirable effects toward environment and the tourism destinations have increased the concern of people involved in it about preservation of the natural resources and long-term preservation of tourism destinations.

The World Commission on Environment and Development (WCED) issued the first report on sustainability which defined the sustainable development as development that "meets the needs of the present without compromising the ability of future generations to meet their own needs". (Choi, Sirakaya, 2005, p.1275). After this report the concept of sustainability was internationalized and universally asked to be applied in all levels of tourism business. But experience has showed that it has not been fully adapted in local levels and that many countries have not clearly implemented policies to support and monitor it.

A special focus is placed upon the sustainable development for the community tourism which according to Choi and Sirakya (2005) is evaluated to be a long- term economic linkage between destination communities and industries and of great importance in improving the lives of the residents and minimizing the negative

1 WTTC March 2021

2 Travel, Tourism and Hospitality - Capital investment in leading countries worldwide on 2019 
effects of tourism on the natural environment. It is of crucial importance in this point, the role of the community managers whose responsibility is to provide information and organize programs for the community stakeholders to raise the awareness on the importance of conservation of the community tourism resources. The last ones should be very actively involved and participate directly in the decision-making process.

According to the literature and recent debates the sustainable tourism involves different dimensions such as ecological, economic, social political technological at the international, national regional and local community levels. It is obvious that these dimensions are interdependent and each of them has its own role to the tourism development.

"Tourism is now so pervasive in modern society that, rather than conceiving tourism as a "departure" from the routines and practices of everyday life, tourism has become an established part of everyday life culture and consumption" (McCabe, 2002:63). Following the above logic, it is evident that tourism is an integral part of the modern life of our society. Moreover, consumer patterns and decision making are very important and has been subject of changes recently. It has been affected considerably from the environment footprint of the tourists themselves too when we find a very important trade-off regarding their preference for unpolluted preserved ecologically areas and destination and its high preference for entertainment and fun at the destination increasing its footprint to these destinations.

Ecological footprint analysis (EF) is evaluated to be a key environmental and effective aggregate indicator of sustainable tourism (ST) that uses gha as the common currency to express impact magnitude across all its components (Hunter and Shaw, 2007, p.46).

First provided in the early to the mid-1990s the EF analysis was first defined from their authors as "an accounting tool that enables us to estimate the resource consumption and waste assimilation requirements of a defined human population or economy in terms of a corresponding productive land area" (Hunter and Shaw, 2007, p.46).

The main attribute of ecological footprint is to provide a powerful educational tool by expressing the demand of natural resources in terms of an equivalent land/sea area by facilitating comprehension of environmental impact. According to Hunter and Shaw, following this logic, it is obvious that EF conceptualizes a population or economy as having "industrial metabolism" which consumes resources and produces wastes to sustain itself by appropriating in this way a portion of the planetary biosphere.

The applications of ecological footprint in tourism are analyzed in the context of environmental impacts on tourist travel mainly and demand upon natural resources to destination areas recently, by attempting to calculate the so called according to 
Hunter (2002), tourism - related EF.

By methodology the net tourism EF is the sum of the components in the transit and destination area less the source country EF for the period away from home generated by the tourist when away from home by leaving on some heating or security lighting1.

The discussion naturally is not limited only to the ecological footprint, even to the total of sustainable tourist indicators, local and global. Here after we shall see an analysis on some factors considered for the pro environmental behavior of the tourists related to their past experiences at the destinations.

\section{Discussion}

The discussion on marketing for quality in tourism, recently has widely taken the route of sustainability, focusing on the modern tourist demand for unpolluted tourist areas and destinations, as well as cultural and historical tourism. We can hold on to this perspective and measure the trend on this regard, finding out the possibility to design the product according to this demand.

The factors possibly affecting this demand and the product design for sustainability and environment preservation and care could be:

- Luxury request of the tourist

- Sports preference during the vacation

- Leisure measured through preferences for fun and entertainment

- Good company at the destination

- The intense experience with the nature at the destination

- The familiar atmosphere at the destination

- Customer's altruist attitude toward environment

- Romantic and nostalgic atmosphere at the destination

- Population density at the destination in top season periods

- Relax space at the destination hotel structure

- Transport pollution (Ecologic footprint)

- Level of acoustic pollution

- Services on site

- Typology of accommodation

1 The procedure for calculating net per capita EF includes five steps in which we must come across the estimates for each indicative (Hunter and Shaw, 2007, p.49). 
- Relationship status of the tourist

- Attractions at the destinations

- Seasonality

- Local community hospitality

- Safeness at the destination

- Obligation and state of order

- Education level

- Gender of the tourist

These factors have a different weight on the customer preferences for tourist products, as well as in their behavior at the destination and their relations to the destination. We can then run a linear multiple regressions on these explanatory variables to measure customers' preference for environmentally sustainable tourist structures and destination using demand for unpolluted tourist areas and destinations or pro-environmental behavior as a dependent variable.

We designed a questionnaire with questions regarding the above listed factors using a Likert scale of five classifications as it is shown in the table below:

The Question Agreement on a five-point- Not scale applicable

$\begin{array}{llllll}5 & 4 & 3 & 2 & 1 & 99\end{array}$

I am interested on luxury rather than taking care on the environment pollution

I like unpolluted surroundings at the destinations

I care about hygiene at the destination

I like sports

I like entertainment and fun

I like doing friendship at the destination

I like of road and nature experiences 
I like familiar treatment at the destination

I have the habit to take care on the tourist environment

I chose romantic destinations

I enjoy high population at the destination

during top season

I like to relax in peaceful places at

destination

I like transport traffic at the destination

I like noisy vacations

I like tourist structures with the largest set

of services on site

I like vacation in family

I lonely individual vacations

I like culture offers at destinations

I like moving during all years to the tourist destinations

I like moving at the top seasons at the tourist destinations

I like hospitality communities at the destinations

I like feeling safe during the holidays at tourist destination

I like obligation, control and state of order at destinations 
I am educated

Gender of tourists counts at environment

preservation

Monthly income in $000 \mathrm{ALL} \quad>100 \quad 70-\quad 50-30-\quad>30$

$100 \quad 70 \quad 50$

We introduced a question on the income level to see the effect of this factor on the total quality of the vacation.

Based on the previous experiences of the interviewed persons at the region of Elbasan during the holiday of the Summer Day on 14th of March 2018, between residents and visitors in sample sizes of 100 people each, many explanatory variables were not significant. We used the backward model selection using the Akaike information criterion (AIC) to eliminate non-significant variables. We resulted at a sample regression model of 7 explanatory variables.

The resulting final model with seven explanatory variables, resulted with a determination coefficient of $0.56\left(\mathrm{R}^{2}\right)$, not very strong but still explaining most of the tourist behavior pro-environment preservation and care. (F-statistic: 8.13 on k=7 and $\mathrm{df}=192$, p-value 0.05), we tested the variables for significance to receive the final sample regression model. It resulted that not all the variables have a strong significance on the dependent variable. This also explains the value of $\mathrm{R}^{2}$. The final regression we run has five principal explanatory variables once tested for significance testifying that their results explained better the friendly pro environmental behavior at the destination. These variables are:

1. I am interested on luxury rather than taking care on the environment pollution (coefficient negative)

2. I like entertainment and fun (negative)

3. I like of road and nature experiences (positive)

4. I like culture offers at destinations (positive)

5. I like unpolluted surroundings at the destinations (positive)

We also analyzed the data on these factors first classifying the customers in three large groups:

1. Small environmental footprint tourists

2. Medium environmental footprint tourists

3. Large environmental footprint tourists 
It resulted that male tourist have a larger ecologic footprint and the first group has a significant share of $38 \%$. Considering the simple moving average method and comparing the data with the international statistics on this regard, we can say that it will result in an increasing trend for unpolluted areas measuring a small environmental footprint in the future.

\section{Target marketing for quality tourism}

This segmentation gives us the possibility to improve the tourist products, as well as to design new tourist products for the future tourist demand in a differentiated way, having differentiated mix for each of the three segments analyzed.

As for the model of tourism to answer to the tourist demand for unpolluted tourist areas and products we chose to use QFD (quality function deployment). It can make possible to deploy tourist product especially those already existing in the market, even in the Albanian market, and chose e part mix of these products to find the ways of improving those by maximization of their effect to the customers related to their pro environmental behavior and perception.

This is an analysis based on performance maximization of the tourist products. To deploy and create the part mix of the tourist product we can use a four-step technique, called HOQ (House of Quality) used by George L. Vairaktarakis ${ }^{1}$ creating four matrices (HOQ) on each step:

1. Voice of the customers to a product's technical requirements - quality deployment based on engineering characteristics and customer requirements

2. Component requirements - parts' deployment based on parts' characteristics and engineering characteristics

3. Manufacturing operations - process planning based on process operations and parts' characteristics

4. Quality control plans - production planning based on quality control and process operations (application of the new standard for competitive advantage)

5. The process is deeply customer oriented and focuses on a customer ranking based on the preferences on the part mix of the presumed tourist product to improve it for a higher performance.

The model used on customer ranking could be:

Customer preferences $=\sum_{k=1}^{n} W_{\mathrm{k}} P_{k l k}\left(C_{k l k}\right)$

and its costs on part mix improvement are $\sum_{k=1}^{n}\left(C_{k l k}\right)$

1 George L. Vairaktarakis, Optimization tools for design and marketing of new / improved products using the house of quality, Journal of Operations Management 17, 1999. pp 645-663 
Where:

$W k$ - weights of the $\mathrm{k}^{\text {th }}$ part improvement

$P k(C k l)$ - performance rating of $p_{k l}$ under $C k l$ costs

$p_{k l}-\mathrm{k}^{\text {th }}$ part of the product

$\mathrm{n}$ - Number of parts in a tourist product

$k$ - Number of parts in a part mix of tourist product

$l$ - lth alternative part choice for $p_{k l}$

Solving the equation using Linear Programming we rank the customers on their preferences to the different alternative part mixes.

While we can model for performance maximization of the tourist product still using QFD adding to the equation a dummy variable (binary one), For $1 \leq k \leq \mathrm{n}_{0}$ and $1 \leq l_{\mathrm{k}} \leq$ $n_{\mathrm{k}}$

introducing it to the model:

$X k l_{k}=\left\{\begin{array}{l}1, \text { if } p \mathrm{klk} \text { is selected among the alternatives for } p \mathrm{k} \\ 0, \text { otherwise }\end{array}\right.$

and

Best-of-class part mix $=(\mathrm{P}) \max \sum_{k=1}^{n o} \quad \sum_{t=1}^{n k} W_{\mathrm{k}} P_{k l k}\left(C_{k l k}\right) X k l$

with these constraints:

$\sum_{i=0}^{n} X k l=1$ for $k=1,2, \ldots, n o$

$\sum_{k=1}^{n o} \quad \sum_{t=1}^{n k} C_{k l k} X k l \leq W$

$X k l_{k} \in\{0,1\}$ for $1 \leq k \leq$ no and $1 \leq l_{\mathrm{k}} \leq n_{\mathrm{k}}$

the constraints (1) correspond to the assignment of the part options in the parts' mix, constraint (2) are budget constraints and constraint (3) corresponds to the integrality constraints. The model of performance (P) maximization is widely acceptable even we introduce a set of parameters which are already calculated and bring to the model the risk of stereotyping of the customer preferences.

The second expression (equation) introduces the probability that the improvement in a specific part increase the performance, giving estimation to each alternative due to relevance of the improvement in a specific part of the product.

Important remarks on the proposed model of performance maximization based on QFD:

- The model used is not very often used

- It concerns both to new product and improved products, but mostly with the 


\section{second ones}

- Gives integrated results on the performance

- Combined effect of the factors considered

- Since we introduce cost constraints, elasticity of demand weights on the deployment process

- The model uses linear programming which is easy to solve but stereotypes costumers increasing ranking and rating errors.

\section{Conclusions}

The study confirms the increasing trend of the orientation of the tourist demand toward unpolluted destinations and attractions, as well as the tendency to safeguard the environment and to use sustainable tourist resources.

It also confirms that the tourists have a strong attraction to turn back to those destinations where their recent or last experience was based on more sustainable tourist activities, both environmental and cultural care based.

It states that a lot of the demand for unsplit tourist destinations is determined considerably by factors related to the total quality of the tourist product, compound by the set of quality services and the destination management in terms of environment care and hygiene and safety at the tourist destination, where the landscape and wildlife play an important role. There are very much appreciated the nature sports too.

The orientation on leisure time is not totally dependent on fun and entertainment, but toward local community life and culture too.

The segmentation of the market due to these important changes on the modern tourist demand recently, ask for selective marketing and product design according to the customer preferences. The model used to optimize and maximize the parts' mix of the tourist product through QFD is a widely good effort and approach to this end.

The model should consider the competition rating too, to design the tourist product, but it belongs to another study and research.

\section{References}

[1] Choi, HwanSuk Ch., Sirakaya, E. Sustainability indicators for managing community tourism. Journal of Tourism Management 27, 2006. pp 1274-1289

[2] George L. Vairaktarakis, Optimization tools for design and marketing of new / improved products using the house of quality, Journal of Operations Management 17, 1999. pp 645-663

[3] Hunter, C., Shaw, J. The ecological footprint as a key indicator of sustainable tourism. Journal of Tourism Management 28, 2007. pp 46-57.

[4] World Travel and Tourism Council, March 2021, https://wttc.org/News- 
Article/Global-TandT-sector-suffered-a-loss-of-almost-US4-trillion-in-2020

[5] Travel, Tourism and Hospitality - Capital investment in leading countries worldwide on 2019 